\title{
Equivalence of Tripartite Quantum States under Local Unitary Transformations
}

\author{
Sergio Albeverio $^{a}{ }^{1}$, Laura Cattaneo ${ }^{a}$, Shao-Ming Fei ${ }^{a, b, c} 3$, Xiao-Hong Wang $^{b} 4$ \\ ${ }^{a}$ Institut für Angewandte Mathematik, Universität Bonn, D-53115 \\ ${ }^{b}$ Department of Mathematics, Capital Normal University, Beijing 100037 \\ ${ }^{c}$ Max Planck Institute for Mathematics in the Sciences, 04103 Leipzig
}

\begin{abstract}
The equivalence of tripartite pure states under local unitary transformations is investigated. The nonlocal properties for a class of tripartite quantum states in $\mathbb{C}^{K} \otimes \mathbb{C}^{M} \otimes \mathbb{C}^{N}$ composite systems are investigated and a complete set of invariants under local unitary transformations for these states is presented. It is shown that two of these states are locally equivalent if and only if all these invariants have the same values.
\end{abstract}

PACS numbers: 03.67.-a, 02.20.Hj, 03.65.-w

Key words: tripartite quantum states, local unitary transformations, entanglement, invariants

Quantum entanglement is one of the most striking features of quantum phenomena [1]. It is playing very important roles in quantum information processing such as quantum computation [2], quantum teleportation [3] (for discussions of experimental realizations see [4]), dense coding [5] and quantum cryptographic schemes [6]. As the degree of entanglement of two parts of a quantum system remains invariant under local unitary transformations of these parts, the invariants of local unitary transformations give rise to an effective description of entanglement. Two states are equivalent under local unitary transformations if and only if they are assigned the same values by all invariants under local unitary transformations. The method developed in $[7,8]$, in principle, allows one to compute all the invariants of local unitary transformations, though in general it is not operational. In [9], the invariants for general two-qubit systems are studied and a complete set of 18 polynomial invariants

\footnotetext{
${ }^{1} \mathrm{SFB}$ 611; IZKS; BiBoS; CERFIM(Locarno); Acc. Arch. USI (Mendrisio) e-mail: albeverio@uni-bonn.de

2e-mail: cattaneo@wiener.iam.uni-bonn.de

${ }^{3}$ e-mail: fei@uni-bonn.de

${ }^{4}$ e-mail: wangxh@mail.cnu.edu.cn
} 
is presented. It is proven that two qubit mixed states are locally equivalent if and only if all these 18 invariants have equal values in these states. In [10] three qubits states are also discussed in detail from a similar point of view. In [11] a complete set of invariants is presented for bipartite generic mixed states.

In this letter, we discuss the locally invariant properties of arbitrary dimensional tripartite quantum states in $\mathbb{C}^{K} \otimes \mathbb{C}^{M} \otimes \mathbb{C}^{N}$ composite systems. We present a complete set of invariants for a class of pure states and show that two of these states are locally equivalent if and only if all our invariants have equal values.

Let $H_{A}$ resp. $H_{B}$ resp. $H_{C}$ be $K$ resp. $M$ resp. $N$ dimensional complex Hilbert spaces. We denote by $\left\{\left|e_{i}\right\rangle\right\}_{i=1}^{K},\left\{\left|f_{i}\right\rangle\right\}_{i=1}^{M}$ and $\left\{\left|h_{i}\right\rangle\right\}_{i=1}^{N}$ the orthonormal bases in $H_{A}, H_{B}$ and $H_{C}$ respectively. A general pure state on $H_{A} \otimes H_{B} \otimes H_{C}$ is of the form

$$
|\Psi\rangle=\sum_{i=1}^{K} \sum_{j=1}^{M} \sum_{k=1}^{N} a_{i j k}\left|e_{i}\right\rangle \otimes\left|f_{j}\right\rangle \otimes\left|h_{k}\right\rangle, \quad a_{i j k} \in \mathbb{C}
$$

with the normalization $\sum_{i=1}^{K} \sum_{j=1}^{M} \sum_{k=1}^{N} a_{i j k} a_{i j k}^{*}=1$ (* denotes complex conjugation).

$|\Psi\rangle$ can be regarded as a state on the bipartite systems $A-B C, B-A C$ or $C-A B$. For each such bipartite decomposition, let us consider the matrix whose entries are the coefficients of the state $|\Psi\rangle$ with respect to the bipartite decomposition. Let $A_{1}$ be the matrix corresponding to $|\Psi\rangle$ as a bipartite state in the $A-B C$ system, with the row (resp. column) indices from the subsystem A (resp. BC). For example, if $K=M=N=2$,

$$
A_{1}=\left(\begin{array}{llll}
a_{111} & a_{112} & a_{121} & a_{122} \\
a_{211} & a_{212} & a_{221} & a_{222}
\end{array}\right) .
$$

Similarly, denoting by $A_{2}$ resp. $A_{3}$ the matrices treating $|\Psi\rangle$ as a state in the $B-A C$ resp. $C-A B$ bipartite system, for $K=M=N=2$ one has:

$$
A_{2}=\left(\begin{array}{llll}
a_{111} & a_{112} & a_{211} & a_{212} \\
a_{121} & a_{122} & a_{221} & a_{222}
\end{array}\right), \quad A_{3}=\left(\begin{array}{llll}
a_{111} & a_{121} & a_{211} & a_{221} \\
a_{112} & a_{122} & a_{212} & a_{222}
\end{array}\right) .
$$

Taking partial trace of $|\Psi\rangle\langle\Psi|$ over the respective subsystems, we have $\operatorname{Tr}_{1}|\Psi\rangle\langle\Psi|=$ $A_{1}^{t} A_{1}^{*}, \operatorname{Tr}_{2}|\Psi\rangle\left\langle\Psi\left|=A_{2}^{t} A_{2}^{*}, \operatorname{Tr}_{3}\right| \Psi\right\rangle\langle\Psi|=A_{3}^{t} A_{3}^{*}$, where $t$ represents the transpose of a matrix. The following quantities are invariants associated with the state $|\Psi\rangle$ given by (1):

$$
I_{\alpha}=\operatorname{Tr}\left(\operatorname{Tr}_{1}|\Psi\rangle\langle\Psi|\right)^{\alpha}, \quad \alpha=1,2, \cdots, S,
$$

where $S=\min \{K, M, N\}$.

In fact, if $\left|\Psi^{\prime}\right\rangle=U_{1} \otimes U_{2} \otimes U_{3}|\Psi\rangle$, with $U_{i}$ unitary matrices acting on the space $H_{i}$, $i=1,2,3$, then $A_{1}^{\prime}$ corresponding to $\left|\Psi^{\prime}\right\rangle$ and $A_{1}$ have the following relation:

$$
A_{1}^{\prime}=U_{1} A_{1}\left(U_{2} \otimes U_{3}\right)^{t}=U_{1} A_{1} V^{t}
$$

where $V=U_{2} \otimes U_{3}$ is also a unitary matrix. So we have

$$
\operatorname{Tr}_{1}\left|\Psi^{\prime}\right\rangle\left\langle\Psi^{\prime}\right|=A_{1}^{\prime t} A_{1}^{\prime *}=\left(u_{1} A_{1} V^{t}\right)^{t}\left(u_{1} A_{1} V^{t}\right)^{*}=V\left(A_{1}^{t} A_{1}^{*}\right) V^{\dagger}
$$


and we get

$$
\operatorname{Tr}\left(\operatorname{Tr}_{1}\left|\Psi^{\prime}\right\rangle\left\langle\Psi^{\prime}\right|\right)^{\alpha}=\operatorname{Tr}\left(V\left(A_{1}^{t} A_{1}^{*}\right)^{\alpha} V^{\dagger}\right)=\operatorname{Tr}\left(A_{1}^{t} A_{1}^{*}\right)^{\alpha}=\operatorname{Tr}\left(\operatorname{Tr}_{1}|\Psi\rangle\langle\Psi|\right)^{\alpha},
$$

i.e., $I_{\alpha}, \alpha=1, \cdots, S$, are invariants.

Similarly, we can construct the following invariants:

$$
\begin{aligned}
& J_{\alpha}=\operatorname{Tr}\left(\operatorname{Tr}_{2}|\Psi\rangle\langle\Psi|\right)^{\alpha}, \quad \alpha=1,2, \cdots, S, \\
& K_{\alpha}=\operatorname{Tr}\left(\operatorname{Tr}_{3}|\Psi\rangle\langle\Psi|\right)^{\alpha}, \quad \alpha=1,2, \cdots, S .
\end{aligned}
$$

There are also other invariants like

$$
\operatorname{Tr}\left(\operatorname{Tr}_{i}\left(\operatorname{Tr}_{j}|\Psi\rangle\langle\Psi|\right)^{\alpha}\right)^{\beta}, \quad i, j=1,2,3, \quad i \neq j, \quad \alpha, \beta=1,2, \cdots, S .
$$

Relevant quantities for states like the Frobenius norm, singular values and the degree of entanglement are all invariants under local unitary transformations. Generally one needs all the invariants to judge whether two tripartite states are locally equivalent. However, for some class of special states, only one kind of invariants, either (2), (3) or (4), is sufficient, as we are going to prove. We first recall some results on matrix realignment [12] and give some definitions.

If $Z$ is an $m \times m$ block matrix with each block of size $n \times n$, the realigned matrix $\tilde{Z}$ is defined by

$$
\tilde{Z}=\left[\operatorname{vec}\left(Z_{11}\right), \cdots, \operatorname{vec}\left(Z_{m 1}\right), \cdots, \operatorname{vec}\left(Z_{1 m}\right), \cdots, \operatorname{vec}\left(Z_{m m}\right)\right]^{t}
$$

where

$$
\operatorname{vec}(A)=\left[a_{11}, \cdots, a_{m 1}, a_{12}, \cdots, a_{m 2}, \cdots, a_{1 n}, \cdots, a_{m n}\right]^{t}
$$

for any $m \times n$ matrix $A$ with entries $a_{i j}$.

It is straightforward to verify that a matrix $U$ can be expressed as the tensor product of two matrices $X$ and $Y$, i.e. $U=X \otimes Y$, if and only if

$$
\tilde{U}=\operatorname{vec}(X) \operatorname{vec}(Y)^{t}
$$

(cf, e.g., [13]).

[Definition]. An $m n \times m n$ unitary matrix $U$ is called unitarily decomposable, if there exist an $m \times m$ unitary matrix $U_{1}$ and an $n \times n$ unitary matrix $U_{2}$, such that $U=U_{1} \otimes U_{2}$.

[Lemma ]. Let $U$ be an $m n \times m n$ unitary matrix. $U$ is a unitarily decomposable matrix if and only if the rank of $\tilde{U}$ is one, $r(\tilde{U})=1$.

[Proof]. Let $U$ be a unitarily decomposable matrix, i.e., there exist unitary matrices $U_{1}$ and $U_{2}$ such that $U=U_{1} \otimes U_{2}$. Applying (7) and using the property that a matrix is rank one if and only if it can be written as product of a column vector and a row vector, we have $r(\tilde{U})=1$.

Conversely, if $r(\tilde{U})=1$, there are matrices $X$ and $Y$ such that $U=X \otimes Y$. On the other hand, due to the unitarity of $U, X$ and $Y$ should satisfy the following equation:

$$
U U^{\dagger}=(X \otimes Y)\left(X^{\dagger} \otimes Y^{\dagger}\right)=X X^{\dagger} \otimes Y Y^{\dagger}=I_{m n}
$$


Let $x_{i j}$ denote the entries of $X X^{\dagger}$. The above relation implies that $x_{i j}=0$ if $i \neq j$ and $x_{i i}=k^{-1} \neq 0, \quad i, j=1, \cdots, m$, and $Y Y^{\dagger}$ is a diagonal scalar matrix, i.e. $X X^{\dagger}=k^{-1} I_{m}$ and $Y Y^{\dagger}=k I_{n}$.

Similarly, we have $X^{\dagger} X=k^{\prime-1} I_{m}$, and $Y^{\dagger} Y=k^{\prime} I_{n}$. It is easily proven that $k^{\prime}=k$. Therefore $X X^{\dagger}=X^{\dagger} X=k^{-1} I_{m}$ and $Y Y^{\dagger}=Y^{\dagger} Y=k I_{n}$. Since $X X^{\dagger}$ and $Y Y^{\dagger}$ are positive and selfadjoint, $k$ is real and positive. Hence $U_{1}=\sqrt{k} X$ and $U_{2}=\frac{Y}{\sqrt{k}}$ are unitary matrices such that $U=U_{1} \otimes U_{2}$ is unitarily decomposable.

Note that if $U=X \otimes Y$ is a unitary matrix, then $X$ and $Y$ are either both unitary or both not unitary.

We can judge whether an $m n \times m n$ unitary matrix $U$ is unitarily decomposable or not in the following way: if the rank of the realigned matrix $\tilde{U}$ is not one, $r(\tilde{U}) \neq 1$, then $U$ is not decomposable. If $r(\tilde{U})=1$, then it can be written as a product of a column vector and a row vector, i.e., there exist $\left(a_{1}, \cdots, a_{m^{2}}\right)^{t}$ and $\left(b_{1}, \cdots, b_{n^{2}}\right)$ such that $\tilde{U}=$ $\left(a_{1}, \cdots, a_{m^{2}}\right)^{t}\left(b_{1}, \cdots, b_{n^{2}}\right)$. These vectors can be obtained from the realignment of certain matrices, say $\operatorname{vec}(X)=\left(a_{1}, \cdots, a_{m^{2}}\right)^{t}, \operatorname{vec}(Y)=\left(b_{1}, \cdots, b_{n^{2}}\right)^{t}$, so that $U=X \otimes Y$. If one of $X$ and $Y$ is unitary, then $U$ is unitarily decomposable.

We consider now the state $|\Psi\rangle$ in (1) as a bipartite state $A-B C$. As shown in [11], two bipartite states $|\psi\rangle=\sum_{i=1}^{M} \sum_{j=1}^{N} a_{i j}|i j\rangle$ and $\left|\psi^{\prime}\right\rangle=\sum_{i=1}^{M} \sum_{j=1}^{N} a_{i j}^{\prime}|i j\rangle$ are equivalent under local unitary transformations if and only if they are assigned the same values for all the invariants: $T_{\alpha}=T_{\alpha}^{\prime}$, for $\alpha=1, \cdots, \min \{N, M\}$, where $T_{\alpha}=\operatorname{Tr}\left(A A^{\dagger}\right)^{\alpha}, T_{\alpha}^{\prime}=\operatorname{Tr}\left(A^{\prime} A^{\prime \dagger}\right)^{\alpha}$, and $A, A^{\prime}$ are the $M \times N$ matrices with the entries $a_{i j}$ and $a_{i j}^{\prime}$ respectively. If $T_{\alpha}=T_{\alpha}^{\prime}$, there exist unitary matrices $U$ and $V$ such that $\left|\psi^{\prime}\right\rangle=U \otimes V|\psi\rangle$, which also implies $A^{\prime}=U A V^{t}$, i.e., $A A^{\dagger}$ and $A^{\prime} A^{\prime \dagger}$ are unitary equivalent and have the same singular values. $U$ and $V$ are dependent on $|\psi\rangle$ and $\left|\psi^{\prime}\right\rangle$, and can be obtained by using the singular value decomposition method: $U=u^{\prime} u^{\dagger}$ and $V=v^{\prime} v^{\dagger}$, where $A=u D v^{\dagger}$ and $A^{\prime}=u^{\prime} D v^{\prime \dagger}$ are singular value decompositions of $A$ and $A^{\prime}$, respectively, with the singular values ordered descending.

Summarizing the above discussions we have the following theorem:

[Theorem]. If two tripartite states $|\Psi\rangle$ and $\left|\Psi^{\prime}\right\rangle$ on $H_{A} \otimes H_{B} \otimes H_{C}$ have the same values of the invariants given by (2), i.e. $I_{\alpha}=I_{\alpha}^{\prime}$ for $\alpha=1, \ldots, S$, there are unitary matrices $U_{1}$ on $H_{A}$ and $V_{1}$ on $H_{B} \otimes H_{C}$ such that $\left|\Psi^{\prime}\right\rangle=U_{1} \otimes V_{1}|\Psi\rangle$. $|\Psi\rangle$ and $\left|\Psi^{\prime}\right\rangle$ are then equivalent under local unitary transformations if $V_{1}$ satisfies $r\left(\tilde{V}_{1}\right)=1$.

[Remark]. If we say that two pure tripartite states $|\Psi\rangle$ and $\left|\Psi^{\prime}\right\rangle$ are a pair of $D_{1}$ states if they satisfy $\left|\Psi^{\prime}\right\rangle=U_{1} \otimes V_{1}|\Psi\rangle$ with $U_{1}$ a unitary matrix on $H_{A}$ and $V_{1}$ a unitarily decomposable matrix on $H_{B} \otimes H_{C}$, we have defined an equivalence relation $\left|\Psi^{\prime}\right\rangle \sim|\Psi\rangle$. Indeed, as $|\Psi\rangle=U_{1}^{\dagger} \otimes V_{1}^{\dagger}\left|\Psi^{\prime}\right\rangle$, where $U_{1}^{\dagger}$ is unitary, and $V_{1}^{\dagger}$ is also unitarily decomposable with $r\left(\tilde{V}_{1}^{\dagger}\right)=1$, one has that if $\left|\Psi^{\prime}\right\rangle \sim|\Psi\rangle$ then $|\Psi\rangle \sim\left|\Psi^{\prime}\right\rangle$. Transitivity also holds, namely, if $\left|\Psi^{\prime \prime}\right\rangle \sim\left|\Psi^{\prime}\right\rangle$ and $\left|\Psi^{\prime}\right\rangle \sim|\Psi\rangle$, then $\left|\Psi^{\prime \prime}\right\rangle \sim|\Psi\rangle$.

We shall provide two examples to illustrate our results.

[Example 1]. We consider two states $|\Psi\rangle=\frac{1}{\sqrt{2}}(|001\rangle+|100\rangle)$, and $\left|\Psi^{\prime}\right\rangle=\frac{1}{\sqrt{2}}(|010\rangle+|111\rangle)$ in $H_{A} \otimes H_{B} \otimes H_{C}$, where $K=\operatorname{dim} H_{A}=2, M=\operatorname{dim} H_{B}=2, N=\operatorname{dim} H_{C}=2$. Let us denote by $\{|0\rangle,|1\rangle\}$ the orthonormal basis of $H_{A}, H_{B}$, and $H_{C}$. We have

$$
\rho=\operatorname{Tr}_{1}|\Psi\rangle\left\langle\Psi\left|=\operatorname{diag}\left(\frac{1}{2}, \frac{1}{2}, 0,0\right), \quad \rho^{\prime}=\operatorname{Tr}_{1}\right| \Psi^{\prime}\right\rangle\left\langle\Psi^{\prime}\right|=\operatorname{diag}\left(0,0, \frac{1}{2}, \frac{1}{2}\right),
$$


and

$$
I_{\alpha}=\operatorname{Tr}\left(\operatorname{Tr}_{1}|\Psi\rangle\langle\Psi|\right)^{\alpha}=\frac{1}{2^{\alpha-1}}, \quad I_{\alpha}^{\prime}=\operatorname{Tr}\left(\operatorname{Tr}_{1}\left|\Psi^{\prime}\right\rangle\left\langle\Psi^{\prime}\right|\right)^{\alpha}=\frac{1}{2^{\alpha-1}}
$$

Since $I_{\alpha}=I_{\alpha}^{\prime}$, we treat $|\Psi\rangle$ and $\left|\Psi^{\prime}\right\rangle$ as states in the bipartite system $H_{A} \otimes H_{B C}$, where $H_{B C}=H_{B} \otimes H_{C}$. Then we get the corresponding $2 \times 2$ block matrices $A_{1}=\left(\begin{array}{cc}T_{1} & 0 \\ 0 & 0\end{array}\right)$, $A_{1}^{\prime}=\left(\begin{array}{cc}0 & T_{1}^{\prime} \\ 0 & 0\end{array}\right)$, where $T_{1}=\left(\begin{array}{cc}0 & \frac{1}{\sqrt{2}} \\ \frac{1}{\sqrt{2}} & 0\end{array}\right), T_{1}^{\prime}=\left(\begin{array}{cc}\frac{1}{\sqrt{2}} & 0 \\ 0 & \frac{1}{\sqrt{2}}\end{array}\right)$. From the singular value decomposition of matrices we have unitary matrices $U_{1}$ in $H_{A}$ and $V_{1}$ in $H_{B} \otimes H_{C}$ such that $\left|\Psi^{\prime}\right\rangle=U_{1} \otimes V_{1}|\Psi\rangle$. In this case $V_{1}=I$. Therefore $|\Psi\rangle$ and $\left|\Psi^{\prime}\right\rangle$ are $D_{1}$ states and they are equivalent under local unitary transformations.

[Example 2]. We consider two states $|\Psi\rangle=\frac{1}{\sqrt{2}}(|110\rangle+|012\rangle)$, and $\left|\Psi^{\prime}\right\rangle=-\frac{\sqrt{6}}{4}|000\rangle+$ $\frac{\sqrt{2}}{4}|010\rangle-\frac{\sqrt{3}}{4}|101\rangle+\frac{\sqrt{3}}{4}|102\rangle+\frac{1}{4}|111\rangle-\frac{1}{4}|112\rangle$ in $H_{A} \otimes H_{B} \otimes H_{C}$, where $K=\operatorname{dim} H_{A}=$ $2, M=\operatorname{dim} H_{B}=2, N=\operatorname{dim} H_{C}=3$. Let us denote by $\{|0\rangle,|1\rangle\}$ the orthonormal basis of $H_{A}$ and $H_{B}$, and by $\{|0\rangle,|1\rangle,|2\rangle\}$ the orthonormal basis of $H_{C}$. We have

$$
I_{\alpha}=\operatorname{Tr}\left(\operatorname{Tr}_{1}|\Psi\rangle\langle\Psi|\right)^{\alpha}=\frac{1}{2^{\alpha-1}}, \quad I_{\alpha}^{\prime}=\operatorname{Tr}\left(\operatorname{Tr}_{1}\left|\Psi^{\prime}\right\rangle\left\langle\Psi^{\prime}\right|\right)^{\alpha}=\frac{1}{2^{\alpha-1}}
$$

Since $I_{\alpha}=I_{\alpha}^{\prime}$, we treat $|\Psi\rangle$ and $\left|\Psi^{\prime}\right\rangle$ as states in the bipartite system $H_{A} \otimes H_{B C}$, where $H_{B C}=H_{B} \otimes H_{C}$; the corresponding $2 \times 6$ matrices $A_{1}$ and $A_{1}^{\prime}$ are, respectively, $\left(\begin{array}{cccccc}0 & 0 & 0 & 0 & 0 & \frac{\sqrt{2}}{2} \\ 0 & 0 & 0 & \frac{\sqrt{2}}{2} & 0 & 0\end{array}\right)$ and $\left(\begin{array}{cccccc}-\frac{\sqrt{6}}{4} & 0 & 0 & \frac{\sqrt{2}}{4} & 0 & 0 \\ 0 & -\frac{\sqrt{3}}{4} & \frac{\sqrt{3}}{4} & 0 & \frac{1}{4} & -\frac{1}{4}\end{array}\right)$. The singular value decomposition delivers us unitary matrices $U_{1}$ in $H_{A}$ and $V_{1}$ in $H_{B} \otimes H_{C}$ such that $\left|\Psi^{\prime}\right\rangle=U_{1} \otimes V_{1}|\Psi\rangle$. For instance,

$$
U_{1}=\left(\begin{array}{cc}
0 & -1 \\
1 & 0
\end{array}\right) \quad \text { and } \quad V_{1}=\left(\begin{array}{cccccc}
1 / 2 & 0 & 0 & \sqrt{3} / 2 & 0 & 0 \\
0 & \sqrt{2} / 4 & -\sqrt{2} / 4 & 0 & \sqrt{6} / 4 & -\sqrt{6} / 4 \\
0 & \sqrt{2} / 4 & \sqrt{2} / 4 & 0 & \sqrt{6} / 4 & \sqrt{6} / 4 \\
\sqrt{3} / 2 & 0 & 0 & -1 / 2 & 0 & 0 \\
0 & \sqrt{6} / 4 & -\sqrt{6} / 4 & 0 & -\sqrt{2} / 4 & \sqrt{2} / 4 \\
0 & \sqrt{6} / 4 & \sqrt{6} / 4 & 0 & -\sqrt{2} / 4 & -\sqrt{2} / 4
\end{array}\right) .
$$

The rank of $\tilde{V}_{1}$ is one, therefore $|\Psi\rangle$ and $\left|\Psi^{\prime}\right\rangle$ are $D_{1}$ states and they are equivalent under local unitary transformations.

[Remark]. We can also say that two pure tripartite states $|\Psi\rangle$ and $\left|\Psi^{\prime}\right\rangle$ are a pair of $D_{2}$ (resp. $D_{3}$ ) states. For example, if we treat $|\Psi\rangle$ as a state in the B-AC system, then $\operatorname{Tr}_{2}|\Psi\rangle\langle\Psi|=A_{2}^{t} A_{2}^{*}$. If $J_{\alpha}=J_{\alpha}^{\prime}$, from the result on bipartite systems we have that $\left|\Psi^{\prime}\right\rangle=$ $U_{2} \otimes V_{2}|\Psi\rangle$, where $U_{2}$ acts on $H_{B}$ and $V_{2}$ on $H_{A} \otimes H_{C}$. If the unitary matrix $V_{2}$ satisfies $r\left(\tilde{V}_{2}\right)=1$, then $|\Psi\rangle$ and $\left|\Psi^{\prime}\right\rangle$ are a pair of $D_{2}$ states and they are equivalent under local unitary transformations. A pair of $D_{3}$ states can be defined in a similar way.

If $|\Psi\rangle$ and $\left|\Psi^{\prime}\right\rangle$ are not a pair of $D_{1}$ states, one can check whether they are a pair of $D_{2}$ or $D_{3}$ states, by using $J_{\alpha}$ and $J_{\alpha}^{\prime}$ or $K_{\alpha}$ and $K_{\alpha}^{\prime}$ to check whether $|\Psi\rangle$ and $\left|\Psi^{\prime}\right\rangle$ are equivalent under local unitary transformations or not.

In summary, we have discussed the local invariants for arbitrary dimensional tripartite quantum states in $\mathbb{C}^{K} \otimes \mathbb{C}^{M} \otimes \mathbb{C}^{N}$ composite systems and have presented a set of invariants 
under local unitary transformations. The invariants are not necessarily independent (they could be represented by each other in some cases), but the invariants are sufficient to judge whether two states constitute a pair of $D_{i}, i=1,2,3$, states, which are equivalent under local unitary transformations.

Acknowledgments The second named author gratefully acknowledges the financial support by the Stefano Franscini Fund. The fourth author gratefully acknowledges the support provided by the China-Germany Cooperation Project 446 CHV 113/231, "Quantum information and related mathematical problems".

\section{References}

[1] A. Peres, Quantum Mechanics: Concepts and Methods, Kluwer, Dordrecht (1993).

[2] See, for example, D.P. Di Vincenzo, Science 270, 255 (1995);

M. Nielsen and I.L. Chuang, Quantum Computation and Quantum Information, Cambridge University Press (2000).

[3] C.H. Bennett, G. Brassard, C. Crépeau, R. Jozsa, A. Peres, and W.K. Wootters, Phys. Rev. Lett. 70, 1895 (1993);

S. Albeverio and S.M. Fei, Phys. Lett. A 276, 8-11 (2000);

G.M. D'Ariano, P. Lo Presti, and M.F. Sacchi, Phys. Lett. A 272, 32 (2000);

S. Albeverio, S.M. Fei, and W.L. Yang, Phys. Rev. A 66, 012301 (2002).

[4] D. Bouwmeester, J.-W. Pan, K. Mattle, M. Eibl, H. Weinfurter, and A. Zeilinger, Nature 390, 575 (1997);

D. Boschi, S. Brance, F. De Martini, L. Hardy, and S. Popescu, Phys. Rev. Lett. 80, 1121 (1998);

A. Furusawa, J.L. Sørensen, S.L. Braunstein, C.A. Fuchs, H.J. Kimble, and E.S. Polzik, Science 282, 706 (1998);

M.A. Nielsen, E. Knill, and R. Laflamme, Nature 396, 52 (1998).

[5] C.H. Bennett and S.J. Wiesner, Phys. Rev. Lett. 69, 2881 (1992).

[6] A. Ekert, Phys. Rev. Lett. 67, 661 (1991);

D. Deutsch, A. Ekert, P. Rozsa, C. Macchiavello, S. Popescu, and A. Sanpera, Phys. Rev. Lett. 77, 2818 (1996);

C.A. Fuchs, N. Gisin, R.B. Griffiths, C-S. Niu, and A. Peres, Phys. Rev. A 56, 1163 (1997).

[7] E.M. Rains, IEEE Transactions on Information Theory 46, 54 (2000).

[8] M. Grassl, M. Rötteler, and T. Beth, Phys. Rev. A 58, 1833 (1998).

[9] Y. Makhlin, Quant. Info. Proc. 1, 243 (2002). 
[10] N. Linden, S. Popescu, and A. Sudbery, Phys. Rev. Lett. 83, 243 (1999).

[11] S. Albeverio, S.M. Fei, P. Parashar, and W.L. Yang, Phys. Rev. A 68, 010313 (R) (2003).

[12] K. Chen and L.A. Wu, Phys. Lett. A 306, 14 (2002).

[13] N.P. Pitsianis, Ph.D. thesis, The Kronecker Product in Approximation and Fast Transform Generation, Cornell University, New York (1997). 